\title{
Advancements in portable and lab based XRF instrumentation for analysis in cultural heritage: A change in perspective
}

\section{Aaron Shugar}

\section{Buffalo State College - SUNY, New York, United States}

Over the last 50 years we have seen tremendous growth in the use of X-ray fluorescence (XRF) in the field of cultural heritage studies. This has expanded in recent years with the desire for non-invasive, non-destructive analysis. Developments in XRF technology have been based on X-ray source, photon detection and computing advancements. This has led to reduced instrument size, and increased photon collection speed. With advanced computer assessment and modeling new avenues of research are being opened for researchers of cultural heritage to explore.

Currently, the proliferation of XRF in cultural heritage studies is widespread, mainly due to the reduced handheld size and ease of use. It has successfully been used on archaeological sites to quickly identify material and guide excavation, in art conservation aiding treatment and research, for analysis of precious gems, geological samples, ceramics, metals, soils to name just a few. New paths of research are revealing themselves with the advent of more powerful computation. The application of machine learning and artificial intelligence algorithms are providing a better understating of the past.

Although the main advancements in X-ray sources have been the shift to X-ray tubes, the reduction of their size, and beam alignment, they have not altered much over time. Major improvements to detectors have had a major effect on the efficacy of portable systems. The shift from Si-Pin detectors to silicon drift detectors dramatically improved count rate from $\sim 20 \mathrm{~K}$ to upwards of $\sim 200 \mathrm{~K}$. This has allowed for faster analytical acquisition time and paved the way for advanced XRF mapping techniques. Detectors are shifting from an $8 \mu \mathrm{m}$ beryllium to a $1 \mu \mathrm{m}$ graphene window, improving light element detection and allows for the detection of elements as low as fluorine.

With higher count rates, smaller aperture collimators can be employed. Although polycapillary systems have spot sizes down to sub $50 \mu \mathrm{m}$, portable systems still use a collimated system and some manufactures are offering apertures down to $1 \mathrm{~mm}$. Current research has shown collimator apertures down to $0.5 \mathrm{~mm}$ is possible with the increased flux while taking advantage of diffraction angles. With these changes, handheld instruments can now be used for more advanced macro area XRF scanning (MA-XRF) and have shown a resolution down to $\sim 0.25 \mathrm{~mm}$ (Shugar, 2020). They have also been used to scan larger fields such as Titian's Rape of Europa, housed at the Isabella Stewart Gardner Museum $(178$ c $205 \mathrm{~cm}$ ) by stitching 40 scans together (Pocobene et al., 2021). With additional image processing, lower resolution scans can be made even sharper (Pouyet et al., 2017). It is in MA-XRF mapping that we have seen a clear change in perspective when dealing with analysis of materials. For example, in the past, researchers asked a specific question that would be answered through spot analysis. For example, differentiating between two pigments in a painting, or determining if heavy metal pesticides remain on ethnographic collections. Now, with the ability to scan larger areas, researchers can change the way they perceive elemental analysis and approach it as an imaging technique. This methodology becomes more powerful when combined with other spectrographic and photographic techniques (i.e. investigating Pollock's number 1 1950: (Dooley et al., 2014)).

With the vast amount of data being produced and the advancement in computer technology, the application of machine learning and artificial intelligence is opening new doors for XRF analysis. Machine learning models such as random forests, gradient boosting, support vector machines and neural networks are all being employed to assess entire spectra and are able to see variation that is not typically witnessed. This has allowed for the 
dating of French gilt bronzes, the identification of moisture levels in soil, and the determination of carbon in geological samples, all through the analysis of XRF spectra.

Machine learning algorithms have successfully been used to improve calibrations as well. Although there are some restrictions and caveats in creating and implementing calibrations, they have shown great promise. Where traditional empirical modeling (e.g., Lucas-Tooth) has been relied upon to create material specific calibration, recent work has focused on the use of several machine learning models to improve those calibrations. Gradient boosting, random forests, neural networks and support vector machines all show excellent results, and in some cases, they can be combined in one calibration for use with different elements (Drake et al., Forthcoming). Testing of archaeological ceramics shows the ability create calibration curves with good cross validation for lighter elements $(\mathrm{Na}, \mathrm{Al}$, and $\mathrm{Si}$ ) even when running instruments at higher voltage setting $(50 \mathrm{kV} 35 \mathrm{uA})$.

These newer models can also be used to classify materials based on qualitative assessment. By looking at the entire spectrum, the models can identify variation in the spectrum that are not traditionally looked for, nor what one can easily discern. The identification of $\mathrm{pH}$ levels in brine, differentiating between dinosaur bones and identification of wood species all had initial success (ibid; Shugar et al., Forthcoming).

Advanced computation is now being employed on larger datacubes from MA-XRF mapping. Using neural networks, self-organizing maps (SOM) can be built and pigments are being defined based on this classification (Kogou et al., 2020).

Smaller, more powerful instruments are undoubtably on their way, allowing for faster analysis and making MA-XRF scanning more affordable. With the implementation of machine learning and artificial intelligence new questions can be asked of material and past analyses can be reinvestigated. The rapid growth of XRF offers a bright future and a clear change in how we perceive XRF analysis of cultural heritage materials.

\section{References}

(1) Shugar, A. N. Handheld Macro-XRF Scanning: Development of Collimators for Sub-Mm Resolution. In Proceedings of the Fourth International Symposium on Analytical MethodsIn Philately; 2020; pp 1320.

(2) Pocobene, G.; Chloros, J.; Shugar, A. N.; Kaiser, B.; Newman, R.; Books, C. Titian's Rape of Europa: Artist's Pigments and Changes Revealed through Macro-XRF Mapping; Jacksonville Florida, 2021.

(3) Pouyet, E.; Devine, S.; Grafakos, T.; Kieckhefer, R.; Salvant, J.; Smieska, L.; Woll, A.; Katsaggelos, A.; Cossairt, O.; Walton, M. Revealing the Biography of a Hidden Medieval Manuscript Using Synchrotron and Conventional Imaging Techniques. Analytica chimica acta 2017, 982, 20-30.

(4) Dooley, K. A.; Conover, D. M.; Glinsman, L. D.; Delaney, J. K. Complementary Standoff Chemical Imaging to Map and Identify Artist Materials in an Early Italian Renaissance Panel Painting. Angew Chem Int Ed Engl 2014, 53 (50), 13775-13779. https://doi.org/10.1002/anie.201407893.

(5) Drake, L. B.; Shugar, A. N.; Kipnis, E. Machine Learning. In Advances in Portable X-ray Spectrometry; Forthcoming.

(6) Shugar, A. N.; Drake, L. B.; Kelley, G. Identification of Wood Species Using XRF and Machine Learning: A Pilot Study. Forthcoming.

(7) Kogou, S.; Lee, L.; Shahtahmassebi, G.; Liang, H. A New Approach to the Interpretation of XRF Spectral Imaging Data Using Neural Networks. X-Ray Spectrometry 2020, 1-10. https://doi.org/10.1002/xrs.3188. 